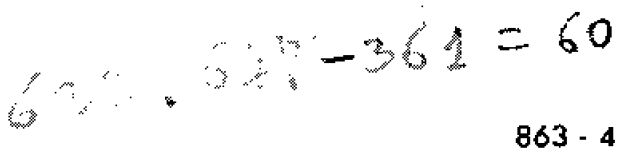

\title{
la mecanización del transporte en una cantera de arcilla
}

GUIDO TORELLI

La mecanización del transporte de los materiales excavados en una cantera de arcilla presenta algunas dificultades que dependen:

- de la naturaleza del terreno que, cuando está fangoso por las lluvias, imposibilita el movimiento de los vehículos;

- del alejamiento continuo del frente de cantera, que impide el empleo de transportadores de tipo corriente, especialmente cuando, a causa de la reducida altura del banco, el alejamiento es muy importante.

En la mayor parte de los casos, el transporte del material desde la canterc al punto de utilización se realiza con vagonetas, procedimiento éste más caro que el efectuodo con transportadores mecánicos continuos.

Es preciso señalor que los transportes realizados por el primer procedimiento requieren mucha mano de obro para mover las vagonetas y llevar a cabo los corrimientos de vías, las cuales deben estar continuomente en las proximidades de las excavadoras y, además, los gastos de conservoción de locomotoras y vagonetas son muy elevados.

Estos gastos resultan notablemente agravados cuando existen pendientes' $y$, todavía peor, cuando es preciso hocer recorridos en cuestas con tracción de vapor.

Con el fin de evitar estos inconvenientes, en la nueva cantera de arcilla de la fábrica de cemento de Spoleto, perteneciente a la TERNI, se ha proyectado y construído una instalación totalmente mecanizada.

El acarreo del material excavado se efectúa con transportadores de cinta. El problema del enlace entre el frente de cantera, que varía frecuentemente, y la parte fija de la instalación, se ha resuelto, de un modo sencillo, mediante un transportador móvil suspendido especial.

La cantera se encuentra en las proximidades de la fábrica y le suministra 250 toneladas diarias de material para hacer el cemento.

El material es una margo arcillosa, cuya característica es la de no ser uniforme; los estratos superiores estón formados por materiales poco compactos, semiplásticos $y$ con un contenido de humedad muy elevado; los inferiores son materiales característicamente margosos, muy compactos y secos. Por requerito el proceso de fabricación, es necesario una perfecta homogeneización; para conseguirlo se le ha dado una gran longitud al frente de cantera, reclizándose la extracción con una excavadora de can" gilones. Esta máquina recorre todo el frente, que tiene cerca de 200 metros, en poco más de una hora y rastrea una capa de $3 \mathrm{~cm}$ de espesor; con tal sistema queda el material suficientemente homogeneizado, ya que; posteriormente, se produce una nueva mezcla durante las operaciones subsiguientes y el secado. 


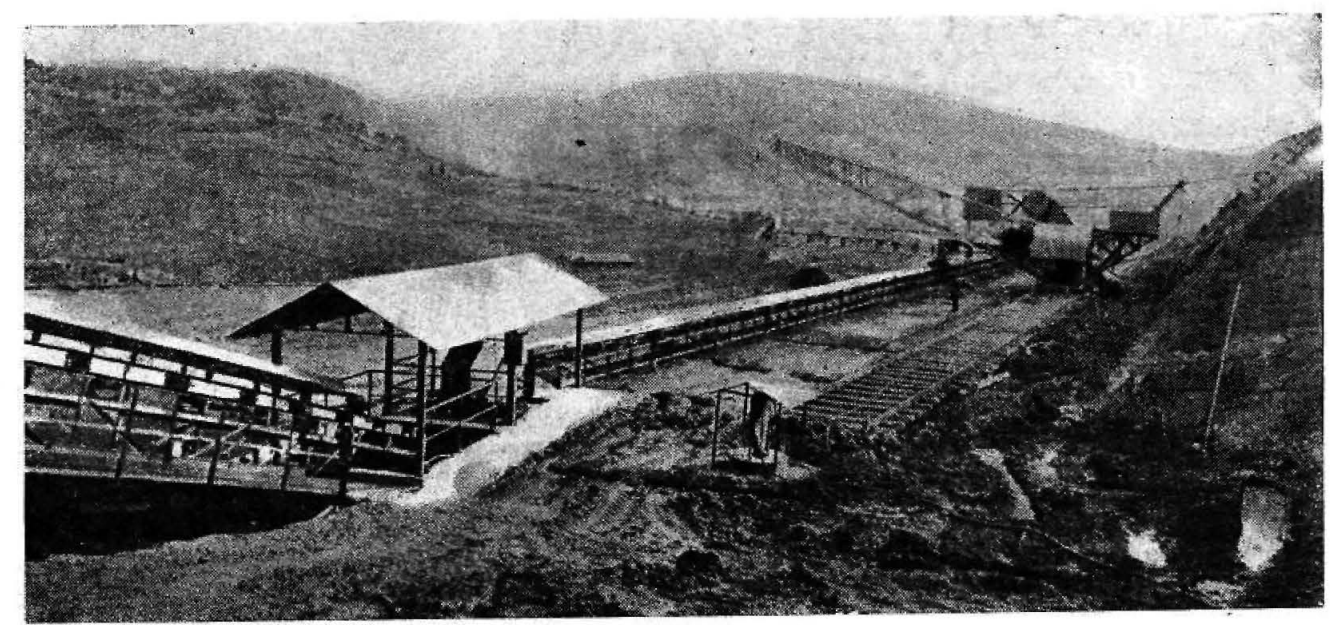

Figura 1

La figura 1 muestra una vista general de la cantera; en la figura 2 puede verse la excavadora de cangilones, y la figura 3 es el transportador de cinta suspendido.

Este transportador, que va montado sobre la excavadora, tiene 30 metros de longitud y pasa por encima de otro transpor-

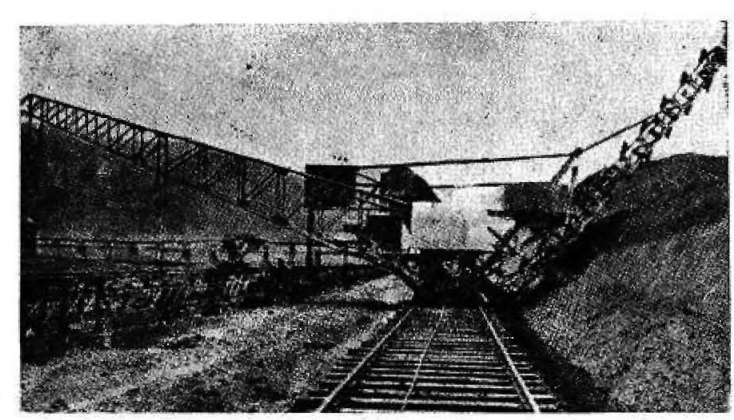

Figura 2

tador de cinta fijo, instalado en dirección ortogonal al primero. Un desviador, que puede variarse de posición (Fig. 3), hace caer el material desde el transportador móvil, que sigue los movimientos de la excavadora, al fijo, que va paralelo al frente de cantera, y que lo conduce hasta el hangar de apilamiento, salvando un desnivel de 20 metros. El material se descarga de la cinta transportadora mediante un carrillo automático, dotado de un dispositivo de lanzamiento para esparcir el material en la fosa del depósito, que tiene 3.000 toneladas de capacidad (Fig. 4).

El material apilado se extrae con una excavadora del mismo tipo que la primera, para transportarlo a la instalación de secado, también mediante un transportador de cinta.

Normalmente el material excavado está constituído por pequeños trozos que, sin ulterior tratamiento, pueden llevarse al secadero.

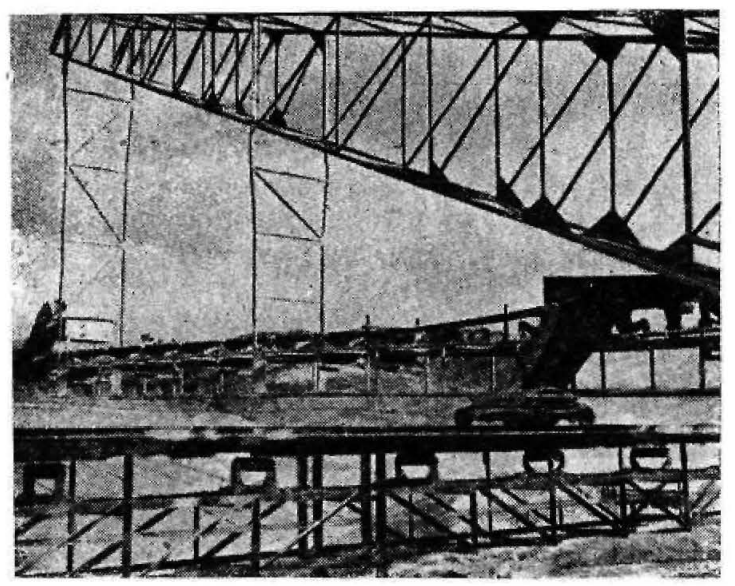

Figura 3 
El material procedente dell banco de arcilla es compacto; a veces se presenta en bloques de cierto peso $(10-12 \mathrm{~kg})$, pero por efecto de impacto, al caer de unos transportadores a otros y por la acción disgregadora del aire se fragmentan, pudiéndose cargar así directamente en el secadero.

La instalación descrita tiene una capacidad de 300 toneladas diarias y requiere muy poca mano de obra.

Periódicamente es necesario trasladar la vía de la excavadora para aproximarla al frente de cantera. Para ello, se precisa una cuadrilla de 6 operarios durante 3 días. Actualmente se efectúa esta operación cada 2 semanos; pero como el frente de cantera va creciendo, en el futuro los desplazamientos se harán a intervalos más amplios.

Se está estudiando una modificación del entramado de la vía, para poder efectuar el desplazamiento de ésta con un número de obreros muy inferior al que actualmente se emplea.

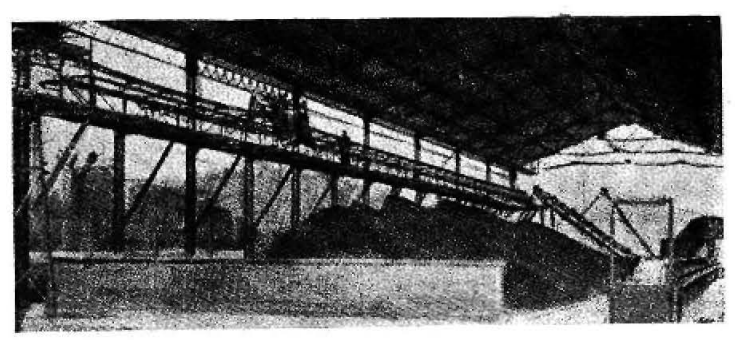

Figura 4

Toda la maquinaria, incluso la excavadora, se mueve con motores eléctricos, y el consumo de energía es de unos 0,5 kWhr. por tonelada de arcilla puesta en el secadero.

Los gastos de conservación son muy reducidos; toda la maquinaria funciona a marcha lenta y el desgaste resultante es muy reducido.

Los motores eléctricos están montados de tal modo, que la parada de cualquier transportador provoca la parada de todos los aparatos de la cantera.

El transportador móvil consta de dos tramos, de 15 metros de longitud cada uno de ellos; en las figuras 1 y 2 puede verse sólo la primera sección.

Dado el enorme peso de este transportador, ha sido necesario montarlo sobre la excavadora. Estas dos máquinas constituyen un conjunto muy pesado.

Cuando el frente de cantera esté alejado 30 metros de la posición inicial, la conexión entre la excavadora y el transportador fijo se conseguirá con una segunda cinta móvil, de la misma longitud que la primera, que correrá sobre una segunda vía.

Más adelante el frente de cantera se alejará más de 60 metros; en este caso, se trasladará la parte del transportador que corre paralelo al frente de cantera y se colocará otro nuevo, transversal, que también será fijo.

El material húmedo, por ser muy adhesivo, se pega en las paredes, especialmente en los puntos de paso de un aparato a otro. Tal inconveniente se ha superado mediante dispositivos adecuados, los cuales eliminan las acumulaciones de material que pueden dar lugar a atascos.

En el transportador móvil y en los que sirven para retirar el material apilado, la cinta es de caucho; en los otros es de acero. Puesto que este material tiene gran tendencia a adherirse, ha sido preciso elegir este tipo de transportador, ya que en ellos resulta fácil la operación de limpiar la cinta.

La instalación se ha estudiado para que la arcilla se encuentre seca al entrar en el ciclo propiamente dicho de la fabricación del cemento, ya que así lo requiere el proceso. Esta misma instalación puede servir para una fábrica de ladrillos, efectuándose las modificaciones que requiera el ciclo de fabricación.

El proyecto de esta instalacion ha sido concebido en la oficina técnica de la fábrica de cemento «TERNI». También se ha ocupado de su realización y puesta a punto.

C. S. C. 